\title{
Elementary Statistical Utensils in Scientific Study Analysis - A Review
}

Ms. Sarabjit Kaur ${ }^{1 *}$, Ms. Simpi Chopra ${ }^{2}$

\footnotetext{
${ }^{1}$ Research Scholar at CTU, Department of Mathematics

${ }^{2}$ Assistant professor at Trinity College, Department of Mathematics
}

DOI: $10.36347 /$ sipms.2021.v08i05.001

| Received: 27.03.2021 | Accepted: 15.05.2021 | Published: 22.05.2021

*Corresponding author: Ms. Sarabjit Kaur

Abstract

Statistical methods complicated in resounding out a study take in development, planning, collecting data, analyzing, illustration expressive understanding and broadcasting of the research findings. The statistical analysis gives importance to the pointless information. Statistical Methods and Tools are back bone of the Research. Statistical tools help for the data analyzing, data description, and forthe all result analysis we use the statistical tool. The greatest part of Statistics is it preserves us informed about what is happening around us. Like mathematics there is no any field in which we are used the application of statistics and its tools. Statistical methods that have comprehensive proposals; however, it does give exceptional helpfulness to statistical methods that are applicable to the financial and social sciences. In calculation to unique research papers, person who reads will find review courses, short notes, intelligences on statisticalsoftware, problematic segment, and manuscript reviews. In this research paper prime focus is the Application of the statistics and its tools. This paper covers the variable, description of the data, ideas of sample size, quantitative variables which are possible only by statistical tools which help in the further researches.

Keywords: Statistic, Statistics Tools, Research, data, Variables.

Copyright $(92021$ The Author(s): This is an open-access article distributed under the terms of the Creative Commons Attribution 4.0 International License (CC BY-NC 4.0) which permits unrestricted use, distribution, and reproduction in any medium for non-commercial use provided the original author and source are credited.

\section{INTRODUCTION}

The word "statistics" is resulting from the Latin word "status", which means political condition or status. Hence, it tracks that the technique of inventory by which a state,' complaint is known called statistics. And is certainty it is through statistics that we find out a state, population and its various sources of income and expenditure Statistics is a part of logical strategy. It achieves the collection, alignment, representation and considerate of information got by investigations and research. The experience in statistics application shows that a solitary factual strategy can be utilized in various exploration regions for managing various kinds of issues. Statistical transcripts cannot be preserved as fictitious texts, since statistical implements are obtainable in a much summarized form (Blalock 1960).

\section{Data collection for Survey and Research:}

Data gathering is one of the maximum important stages in directing a research. We can have the best research design but if we cannot collect the required data we will not be able to complete our project. Data collection is an organized progression of assembly explanations or quantities. Whether we are conducting research for business or academic purposes.
Data collection for investigations and research helps in decision making and preserves the truthfulness of research. Before introducing various types of data collection its' important to understand that data collection in itself falls under two broad categories; Primary data collection and secondary data collection.

\section{Primary Data Collection}

Primary data collection is the gathering of raw data collected at the source. It is a procedure of assembling the innovative data composed by a researcher for a definite research determination. It can be collected in number of ways. Primary data gathering is relatively exclusive and phase overshadowing.

\section{Secondary Data Collection}

Secondary data collection is the collection of data by an individual who is not the original user. It is the process of collecting data that is already existing or already published books, journals and/or online portals. Secondary data collection is less expensive and easier to collect.

Data collection is further categorized in two categories: Qualitative \& Quantitative. 


\section{Quantitative Data}

Quantitative data is about collecting and analyzing data to explain the phenomena. It is numerical in nature and can be mathematically calculated. Quantitative data can be analyzed through various statistical methods like mean, median, mode, regression, correlation etc. It can be expressed graphically through various means like bar graph, pie chart, histogram or scatter graph etc.

There are several methods through which we can collect quantitative data. Which includes

1. Probability sampling

2. Observations

3. Surveys

4. Interviews

5. Document review

Probability sampling: Probability selection is definite as a collection technique in which the researcher indicates models from a higher population using a process based on the concept of probability. For a applicant to be particular as a probability sample, he/she must be nominated using a random selection.

The supreme serious constraint of probability sampling is that everybody in your population has a known and equivalent unplanned of receiving selected. For example, if you have a population of 100 people, every individual would have probabilities of 1 in 100 for receiving nominated. Probability sampling gives you the optimum unplanned to generate a sample that is surely illustrative of the population.

Probability sampling uses statistical theory to unsystematically choice a minor collection of people (sample) from an existing huge population and then forecast that all their responses will competition the inclusive population.

Observations: Quantitative statement is an independent assembly of data which is fundamentally engaged on facts and values it suggests "connected to, of or represented in terms of a measure". Results of quantitative statement are consequential using statistical and numerical analysis methods. It indicates observation of any entity that can be associated with a numeric value such as age, shape, weight, volume, scale etc. This estimation technique is directed on a sample which best represents the goal market. It is important to have a superior sample size so that the interpretations can be made considering most of the varieties that exist in a population. By considering a large population, the observation results are most likely to have advanced credibility.

Surveys: A survey is a enquiry method used for assembling facts from a predefined cluster of respondents to expansion material and perceptions into innumerable topics of interest. They can have multiple determinations, and researchers can behavior it in many ways provisional on the organization preferred and the study's objective. In the year 2020, research is of exciting significance, and hence it's important for us to recognize the assistances of social research for a board population using the right survey tool.

The facts is usually attained through the practice of homogenous processes to certify that each respondent can answer the enquiries at a level playing field to avoid biased opinions that might impact the consequence of the exploration or study. The process involves asking individuals for information comprehensive a questionnaire, which can be either online or offline. However, with the appearance of original technologies, it is common to allocate them using alphanumeric media such as social networks, email, QR codes, or URLs.

Interviews: In a structured interview, the researcher asks a standard set of questions and nothing more. (Leedy and Ormrod, 2001)

Face -to -face interviews have a separate improvement of allowing the researcher to found understanding with possible contributors and therefore improvement their collaboration. These interviews produce premier response duties in survey research. They correspondingly permit the researcher to explain uncertain answers and after suitable, pursue follow-up information. Disadvantages contain unrealistic when huge illustrations are complicated time consuming and expensive. (Leedy and Ormrod, 2001)

Telephone interviews are less time consuming and less exclusive and the researcher has complete admittance to someone on the planet who has telephone. Disadvantages are that the reaction amount is not as high as the face-to- face interview as but significantly sophisticated than the transmitted survey. The trial may be influenced to the magnitude that individuals without receivers are part of the population approximately whom the researcher requirements to attraction interpretations.

Computer Assisted Personal Interviewing (CAPI): It is a procedure of individual interviewing, but in its place of implementation a opinion poll, the investigator carries lengthways a processer or hand-held processer to arrive the evidence straight into the catalogue. This technique protects stretch complicated in dispensation the facts, as well as equivalent the questioner from stentorian everywhere hundreds of surveys. However, this category of facts gathering technique can be exclusive to set up and needs that examiners have processer and inputting services. 


\section{Questionnaires: Types of questionnaires are as following}

Paper-pencil-questionnaires: can be directed to a huge numeral of persons and protects the scholar time and money. Individuals are supplementary straightforward while answering to the surveys regarding controversial subjects in specific due to the detail that their responses are unspecified. But they also have disadvantages. Mainstream of the individuals who receive surveys don't return them and those who do strength not be illustrative of the formerly nominated illustration (Leedy and Ormrod, 2001).

Web based questionnaires: A original and certainly mounting organization is the usage of Internet founded investigation. This would unpleasant getting an e-mail on which you would click on an speech that would take you to a protected web-site to fill in a questionnaire. This category of investigation is frequently earlier and fewer comprehensive. Certain difficulties of this technique comprise the prohibiting of persons who do not have a computer or are incapable to access a supercomputer. Also the validity of such surveys is in question as people might be in a hurry to complete it and so might not give truthful responses.

Questionnaires frequently kind use of Specification and evaluation scales. These strategies help make simpler and quantify people's performances and insolences. A checklist is a list of performances, characteristics, or other entities that researcher is looking for. Both the investigator and survey contributor simply checks whether each item on the list is experiential, current or true or vice versa. A rating scale is further useful when a performance requirements to be appraised on a range. They are also known as Likert scales. (Leedy and Ormrod, 2001).

Qualitative Data: Qualitative data is expressed in words and is non-numerical. This means Qualitative data collection is often in the form of words or sentences. Such type of data includes feelings, emotions or subjective perception of something. Qualitative questions are open-ended. It includes focus group discussions and interviews.

\section{VARIABLES AND ITS TYPES}

Variable: Variable is a typical that varies after one separate associate of population to additional separate member. Variables such as height, weight are dignified by specific kind of scale and originates below measureable variables. Sex and eye color originates below qualitative material and are called qualitative variables.

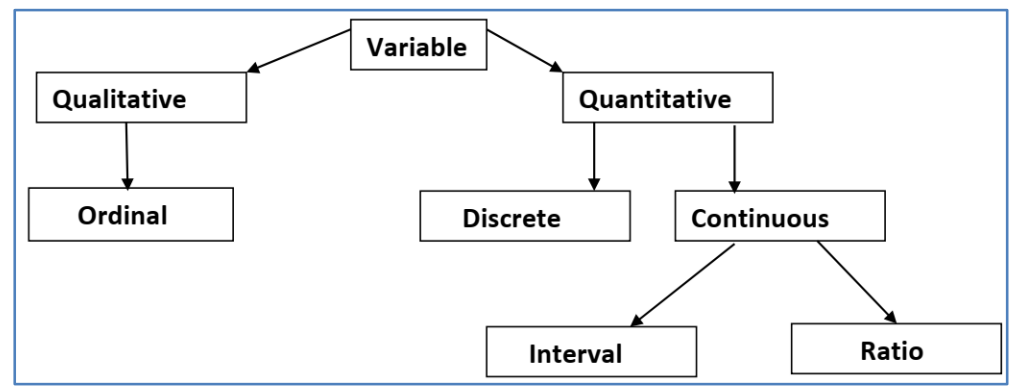

Nominal Scale: A nominal scale is one in which matters or entities are allocated to groups that have no mathematical possessions. Nominal scales have the typical of individuality but lack the additional possessions. Variables measured on a nominal scale are frequently mentioned to as unconditional variables because the measuring scale includes separating the data into groups. However, the groups transmit no arithmetical mass. Certain examples of unconditional variables, or facts measured on a nominal scale, are ethnicity, gender, and political association. We can allocate numerical standards to the levels of a nominal variable.

Ordinal Scale: an ordinal scale, matters or entities are characterized, and the groups form a rank order along a range. Data stately on an ordinal scale have the possessions of individuality and amount but lack equal component size and complete zero. Ordinal data are frequently mentioned to as hierarchical facts because the facts are ordered from maximum to lowermost, or major to minimum.

Interval Scale: In an interval scale, the components of measurement (intervals) between the statistics on the scale are all equivalent in magnitude. When you usage an interval scale, the conditions of uniqueness, scale, and identical unit scope are happened.

Ratio Scale: In a ratio scale, in calculation to instruction and identical elements of dimension, an complete zero specifies an time off of the variable being measured. Ratio data have all four possessions of measurement - identity, magnitude, equal unit size, and absolute zero. Samples of proportion weighing machine of dimension comprise weight, time, and height.

\section{Discrete and continuous variables}

Additional means of categorizing variables is in relations of whether they are discrete or continuous 
Sarabjit Kaur \& Simpi Chopra., Sch J Phys Math Stat, May, 2021; 8(5): 88-95

in nature. Discrete variables frequently contain of complete quantity elements or groups. They are made up of chunks or units that are disconnected and different from single alternative. A modification in value happens a complete unit at a time, and decimals do not kind intellect with distinct scales. Greatest nominal and ordinal data are discrete. For illustration, sex, partypolitical party, and civilization are discrete scales. Certain intermission or ratio facts can be discrete. For example, the amount of children somebody has is stated as a entire amount (discrete data), yet it is also ratio data (you can have a true zero and form ratios). Continuous variables frequently reduction lengthways a range and permit for slight quantities. The term continuous means that it "continues" among the whole number units. Examples of continuous variables are stage (22.7 years), tallness (64.5 inches), and mass (113.25 pounds). Maximum intermission and ratio facts are continuous in nature. Discrete and continuous data will become more important in advanced episodes when we discuss investigation project and facts performance.

\section{Measures of Central Tendency}

Measures of central Tendency is most common and easy way for the description of the data in the any research analysis. Frequency dispersal organizes the heap of data into a few significant groups. Collected data can also be summarized as a on its own index/value, which characterizes the complete data. These procedures may also support in the assessment of statistics. CENTRAL TENDENCY. It is the only significance that is best typical/ characteristic of the composed data. The duration "number crunching" is castoff to clarify this characteristic of statistics explanation. The mean, median and mode are the three frequently used procedures of central tendency. Measures of Central Tendency also delivers the tackles who help to division the statistics according to the Mathematical Measures and Positional Measures.

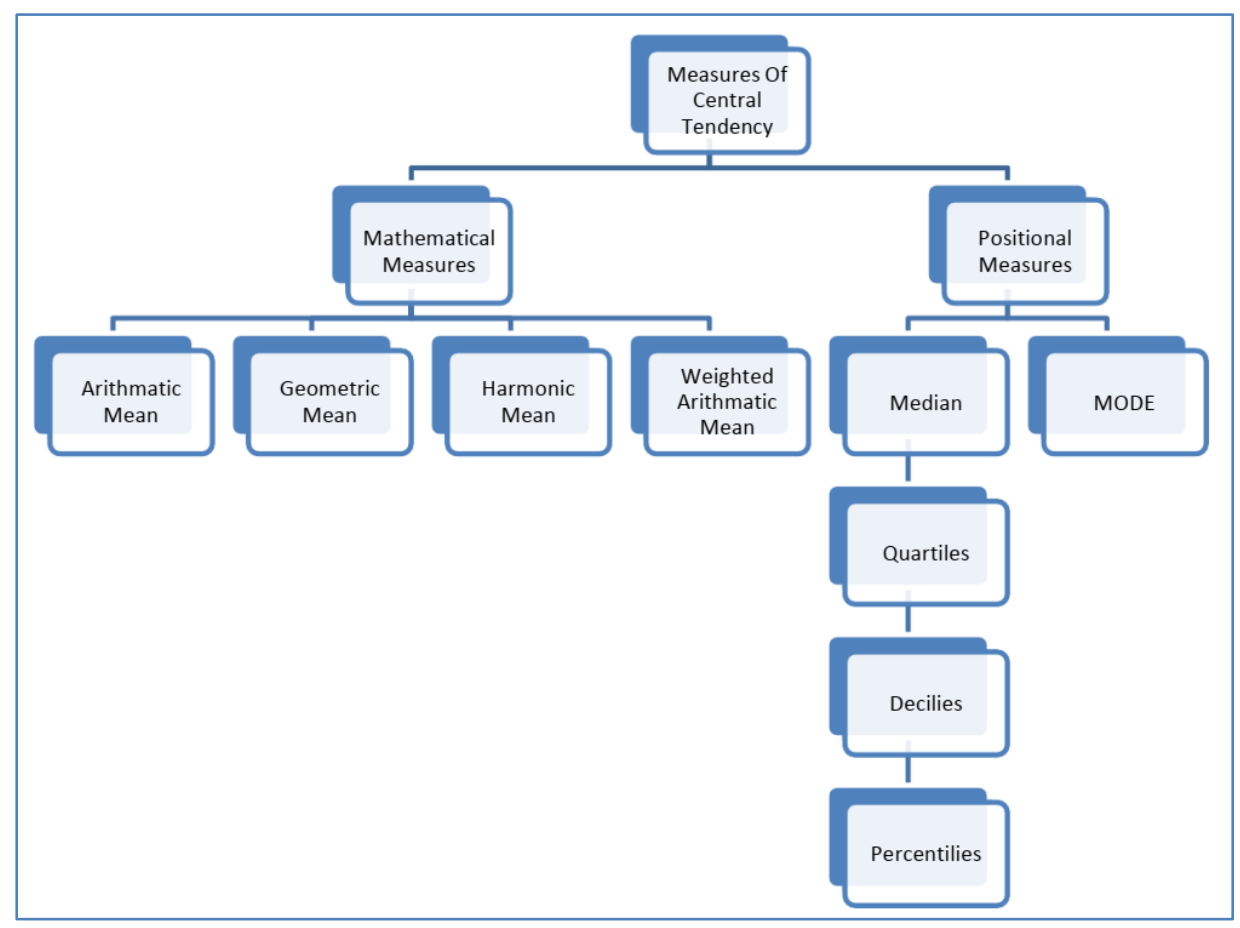

Arithmatic Mean: Arithmetic mean is the only averages which qualify all the essential requisites of the good measure of central tendency. It is also called common average. Each and every item has equal share in determining the arithmetic mean." Arithmetic mean is a numerical value obtained by adding all the terms or value of the series and dividing the total by the numbers of the terms."

$$
\overline{\mathrm{X}=} \frac{\Sigma X}{n} \text { In different types of the series }
$$
individual, discrete and continuous series there are different Method to apply the methods of mean. Arithmetic mean has also facilities for the further algebraic treatment which is very useful in the research.
Geometric Mean: Geometric mean is another mathematical measure which helps to find the nth root of the product of the observation. Geometric mean is also help to fund the averages growth rate in increasing and decreasing form in the Research analysis. It is very important tools of central tendency which used in management, marketing, business. Symbolically it is representing in the form of

$$
\mathrm{G} . \mathrm{M}=n \sqrt{ } \prod x ; 1 \leq \mathrm{i} \leq \mathrm{n}
$$

G.M is most suitable average while dealing with data pertaining to economic and social changes. It is most appropriate average for calculation of index numbers. 
Harmonic Mean: Harmonic is the basically reciprocal of the AM symbolically it is defined by H.M $=\frac{n}{\sum 1 / x}$

$\sum 1 / x$ H.M is also helpful for when terms are given form of negative value. It also hep to find Unknown parameter when two or three are given

Weighted Arithmetic Mean: Weighted Arithmetic mean useful for the variety of the data, different values of the series have different importance. It is very useful when groups divided into subgroup and subpart. It gives the importance to all the items.

$$
\mathrm{Xw}=\frac{\sum W X}{\sum W}
$$

Median: Median is the one of the positional Measures.it help to divide the data into two equal distribution.it is generally denoted by M. "Median is the value which separates the data into two same sizes which gives the one value more effectible as compare to other value". Median can be calculated graphically making use of give. It is least affected by extreme values in the series. It is also called the central value of the distribution

Other Positional Measures (Quartiles, Deciles, Percentiles)

Median divides the given data into two exactly two equal parts, when it has been arranged in the ascending and descending order of magnitude. Apart of median there are other positional measures which are also known as partition values or fractiles. Quartiles help to divide the distribution into for equal parts. Deciles divide the data into ten equal parts when data arranged. Each decile shows $10 \%$ of the total numbers of items. D5 is equal to median. Percentiles help to divide the data into hundred equal parts, the points of division are known as percentiles. Each part is equivalent to $1 \%$ of the total number if items and P50 is equal to Median.

\section{Quartiles}

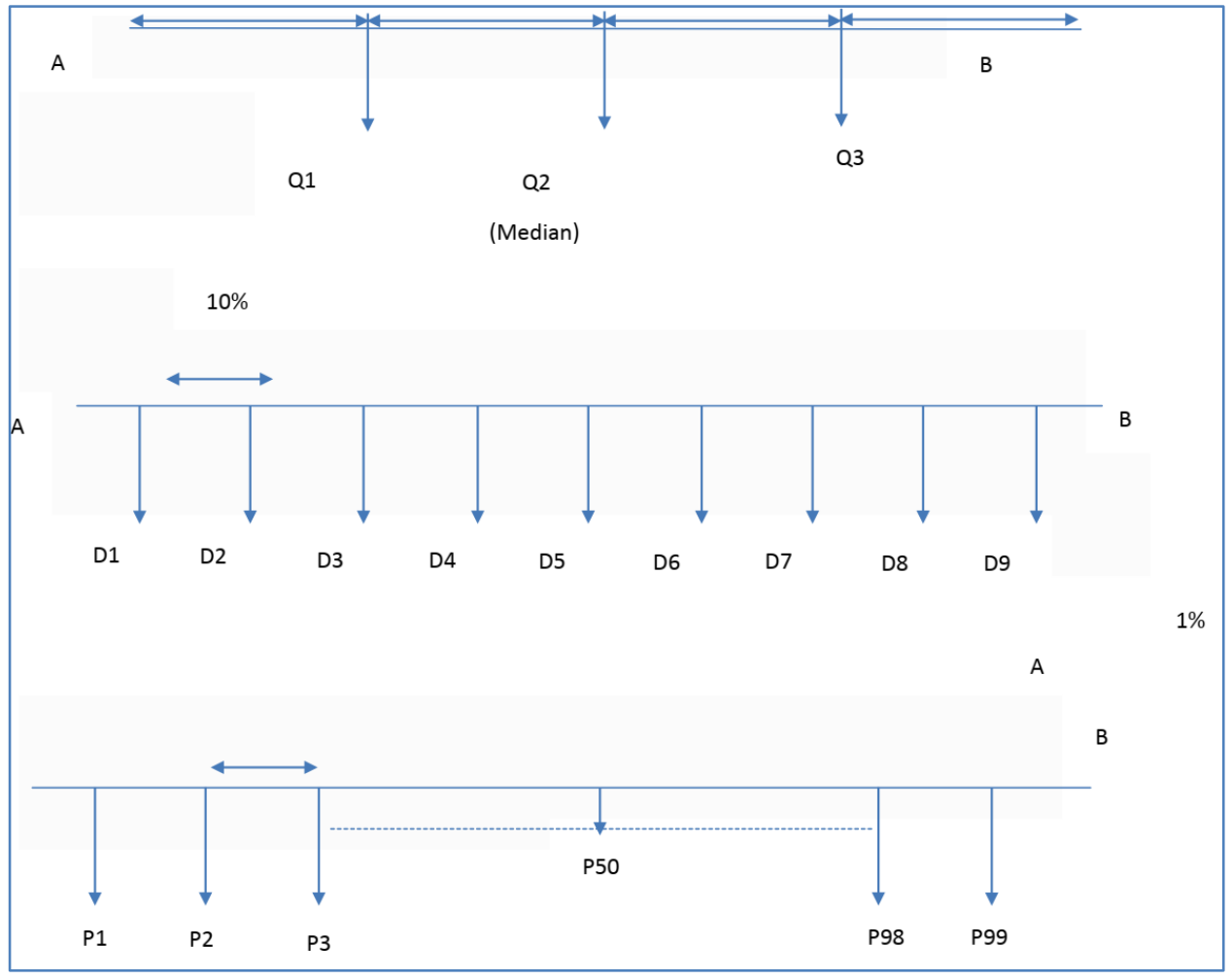

Ode: The most occurrence value in the distribution is called Mode. It is generally denoted by $\mathrm{Z}$. Mode is that assessment of the variable about which there is maximum attentiveness of values. It is additional positional measure of central tendency. "The value of the variable which occurs most frequently in a distribution is called the mode" mode has further model Bi model, Uni Model, Tri Model which help is the further treatment of the distribution. A very important application of the mode that use in Commercial and
Scientific use. Mode is used in studying Qualitative Phenomena.

Measures of Dispersion: Dispersion is an important statistical technique with the help of which we study the variability in a given series about its central and mean value. Measures of variation help to examine the reliability of the averages and also compare the series on the basis of variability. Range, Mean Deviation, Standard Deviation, Quartile Deviation are 
Sarabjit Kaur \& Simpi Chopra., Sch J Phys Math Stat, May, 2021; 8(5): 88-95

different form of Measures of dispersion. Mean Deviation and Standard Deviation are used for Graphic variation and other forms are used for absolute Measures and Relative Measures. Standard deviation is used in statistical tools like skewness, correlation, regression, analysis of variance and testing of hypothesis.

Range describes the range, or variability, of an example. It is designated by the smallest and supreme values of the variables. If we rank the data and after ranking, collection the explanations into percentiles, we can get improved material of the pattern of spread of the variables. In percentiles, we rank the explanations into 100 identical parts. We can then define 25\%, 50\%, 75\% or any additional percentile quantity. The median it he 50th percentile. The interquartile variety will be the explanations in the central $50 \%$ of the explanations about the median (25th-75th percentile). Variance is a measure of how spread out is the spreading. It gives a suggestion of how adjacent and separate statement groups about the mean value. The variance of inhabitants is definite by the resulting formula:

$$
\sigma^{2}=\frac{\sum_{i=1}^{N}(X i-X)^{2}}{N}
$$

Where $\sigma^{2}$ is the population variance, $\mathrm{X}$ is the population mean, $\mathrm{Xi}$ is the ith element from the population and $\mathrm{N}$ is the number of elements in the population. The variance of a sample is defined by slight

\section{Correlation: meaning and coefficient}

Correlation is intended for discovering the grade of connection between two variables in consideration. Correlation coefficient is the quantity to measure such degree of relationship of the variables. Usually, two correlation coefficients are secondhand in applications, specifically: Pearson's Product Moment Correlation Coefficient and Spearman's Rank Correlation Coefficient. This paper chiefly reflects the presentations of Pearson's Simple Linear Correlation in discovering the relationship among variables.

In 1896, correlation coefficient is first expressed and discovered by Karl Pearson (Hauke and Kossowski, 2011), with the thoughts of correlation by Francis Galton and the qualified influence by Auguste Bravais (Denis, 2001). Hauke and Kossowski (2011) do approve that the Pearson's 1 Correlation by measure can be two types: Simple Correlation and Multiple Correlation (Partial and Total). Also, correlation can be linear of non-linear. Product Moment Correlation Coefficient ( $\mathrm{R}$ or $\mathrm{r}$ ) is a rule to quantity the strength of direct suggestion among the variables.
As it procedures the degree of linear suggestion of variables, intermission or relationship variables should be in reflection with a situation that the variables measured should reduction in normal distribution.

Pearson's mathematical formulation to quantify the degree of relationship (R) between variables, namely, $\mathrm{X}$ and $\mathrm{Y}$, can be given as:

$R=n\left(\sum X Y\right)-\left(\sum X\right) \cdot\left(\sum Y\right)$
$\sqrt{\left(\sum X^{2}\right)-\left(\sum X\right)^{2} \sqrt{ } n\left(\sum Y^{2}\right)-\left(\sum Y\right)^{2}}$

Where, $\mathrm{n}=$ Number of observations $\mathrm{x}=$ Measures of Variable 1

$\mathrm{y}=$ Measures of Variable 2

$\Sigma x y=$ Sum of the product of respective variable measures $\Sigma x=$ Sum of the measures of Variable 1 $\Sigma y=$ Sum of the measures of Variable 2

$\Sigma \mathrm{x}^{2}=$ Sum of squared values of the measures of

Variable $1 \Sigma y^{2}=$ Sum of squared values of the measures of Variable 2

If the tendency of a variable is optimistic and virtually comparable to additional variable, there may be opportunity to have optimistic suggestion of each other and such suggestion can deliver optimistic correlation coefficient; and If the tendency of a variable is optimistic and nearly destructive to additional variable, there may be opportunity to have destructive suggestion of each other and such association can result in destructive correlation coefficient. Essentially, the coefficient of correlation $\mathrm{R}$ will variety between -1 and +1 , i.e., $-1 \leq \mathrm{R} \leq+1$. There is no a definite technique of understanding the correlation coefficient. According to Gogtay and Thatte (2017), by quantity, the correlation coefficient can be understood founded on its value

\section{Simple regression}

If relationship among two (one dependent and other independent) variables is considered at a period then the regression is called simple, whereas the training of more than two variables at a time is multiple. If $\mathrm{Y}$ is one of the dependent variable and $\mathrm{X}$ is an its independent variable then regression equation of $\mathrm{Y}$ is

$\mathrm{Y}=\mathrm{a}+\mathrm{b} \mathrm{X}$ Where, $\mathrm{a}=\mathrm{y}$ intercept $=$ constant $=$ value of $\mathrm{Y}$ when $\mathrm{X}=0$

$\mathrm{b}=$ regression coefficient $=$ slope coefficient $=$ change in the value of $Y$ per unit change in the value of $X$.

Multiple Regressions: Let ' $y$ ' is one of the dependent variable and $\mathrm{a} 1, \mathrm{a} 2, \mathrm{a} 3$. Ak be the ' $\mathrm{k}$ ' independent variables. Then the multiple regressions typical is defined as

$\psi=\beta 0+\beta 1 \xi 1+\beta 2 \xi 2+\ldots \ldots \ldots \ldots \ldots \ldots \ldots+\beta \kappa \alpha \kappa+\varepsilon$

Where, $\mathrm{y}=$ dependent variable and $\mathrm{a} 1, \mathrm{a} 2, \mathrm{a} 3 \ldots \ldots \ldots \mathrm{.ak}$ are independent variables. $0=\mathrm{y}$-intercept. 
Sarabjit Kaur \& Simpi Chopra., Sch J Phys Math Stat, May, 2021; 8(5): 88-95

$1=$ Incline of $y$ with variable a1 holding the residual variables a2, a3 ...,ak constant or Regression coefficient of $y$ on a1 possessions the residual variables a2, a3 ............ ak constant. And so on. (Dendukuri \& Reinhold, 2005)

Some pre-requisites to transport linear regression model are

- There is linear relationship between measureable dependent and independent variables

- There is no occurrence of autocorrelation of residuals.

- The mean of residuals is zero.

- There is identical variance of residual or existence of homoscedasticity.

- The independent variables are uncorrelated with errors

- $\quad$ There is absence of multicollinearity. (Zaid, 2015)

\section{SPSS}

SPSS mentions to Statistical Platform for Social Science. It is statistical software which facilities to collect and examine data. We can collect or access collected prime data or minor as same as Microsoft Excel. Its menu bar is supportive to analyze the statistics thus arrived simply. Various statistical investigations can be approved using SPSS (Arkkelin, 2014). Various researchers have functional the correlation and regression analysis in their thesis, courses and their papers, however; they are not yet assertive for the suitable practice of correlation and regression analysis and how to appropriate these statistical tools in their research entirety. In some cases, their explanation may deceive their research studies. Many beginner researchers are enthusiastic to procedure correlation and regression investigation but they don't know how to practice these implements during their statistics investigation. The primary independent of this study is to portion information on regression and correlation investigation and essential surroundings to practice in their research paper.

\section{Factor Analysis}

The outcomes from a factor analysis of a correlation matrix and the consistent covariance matrix are not identical. When considering a covariance matrix, variables having large variance will stimulus the results of the analysis supplementary than will variables having minor variance. Factor analysis has been charity in two data analytic situations: in an assenting routine considered to authorize or disprove the theorized construction, or to try to discover a structure, in which circumstance the examination is called exploratory.

\section{Uses of factor Analysis in Research}

The subsequent are the presentations of Factor Analysis appropriate to a number of systematic and strategy apprehensions.

\section{Interdependency and pattern delineation}

Factor Analysis may be used to recognize the linear relationships into their dispersed arrangements. Each arrangement will seem as a factor describing a separate cluster of interconnected statistics.

\section{Data Reduction}

It can be useful for reducing a quantity of material to inexpensive explanation.

\section{Structure}

Factor Analysis may be working to determine the uncomplicated construction of a sphere.

\section{Classification or description}

It is an instrument for emerging an experiential typology. It can be used to collection interdependent variables into expressive groups, such as thought, revolution, generous elective, and oppression. It can be used to categorize country summaries into categories with comparable appearances or performance.

\section{Scaling}

The scale may mention to such occurrences as administrative contribution, elective performance, or conflict. Factor Analysis suggestions an explanation by separating the appearances into independent foundations of deviation (factors). Every influence then characterizes a scale created on the experimental connection between the individualities.

\section{Hypothesis testing}

There are abundant suggestions concerning magnitudes of approach, disposition, collection, common behavior, elective, and battle. Subsequently the significance typically connected with 'dimension' is that of a collection or group of extremely inter associated appearances or performance, Factor Analysis may be used to examination for their experimental presence. Which 17 characteristics or performance should, by philosophy, be associated to which proportions can be hypothesized in improvement and arithmetical tests of implication can be functional to the FA results.

Moreover those involving to magnitudes, there are supplementary varieties of suggestions that may be confirmed e.g. if the concern is with a association between commercial improvement and variability, property other belongings continuous, a Factor Analysis can be done of economic and instability variables along with other variables that may affect (hide, intermediate, decrease) their relationship. The subsequent influences can be so well-defined (rotated) that the first numerous influences comprise the intermediating procedures (to the maximum allowed by the empirical relationships).A outstanding independent influence can be considered to greatest define the hypothesized associations among the commercial and variability procedures. The degree of connection of both variables in this arrangement 
Sarabjit Kaur \& Simpi Chopra., Sch J Phys Math Stat, May, 2021; 8(5): 88-95

qualifies the researcher to see whether and commercial improvement variability arrangement essentially happens when additional effects are believed continuous.

\section{Data transformation}

Factor Analysis can be used to change statistics to happen the supposition of supplementary procedure for illustration presentation of the various regression techniques assumes (if experiments of consequence are to be functional to the regression quantities) that predictors -the so- called independent variables - are statistically unconnected. If the analyst variables are associated in defilement of the supposition, Factor Analysis can be working to diminish them to a minor usual of uncorrelated influence scores. The scores might be used in the regression investigation in residence of the unique variables, with the information that the expressive variation in the unique statistics has not been misplaced. Likewise, a huge quantity of dependent variables can also be concentrated through Factor Analysis.

\section{Exploration}

The unidentified field may be discovered through Factor Analysis. It can diminish compound inter relationships to a comparatively unassuming linear appearance and it can discover unpredicted, possibly surprising, associations.

\section{Mapping}

Besides simplifying examination Factor Analysis also permits a researcher to diagram the community situation. Mapping means the methodical effort to diagram main experimental perceptions and foundations of dissimilarity.

\section{CONCLUSION}

After discussing the tools of statistics we can say that without statistics tools research can never be possible without statistics we would be incompetent to tease apart the multitude of possessions that may be manipulating our reliant on variable. It is significant that an investigator knows the impressions of the elementary numerical methods used for conduct of investigation training. This wills assistance to conduct a suitably well-made study leading to effective and dependable results. Unsuitable procedure of statistical procedures may central to defective conclusions, positive miscalculations and undermining the consequence of the object. Immoral statistics may central to bad research, and bad investigation may lead to unprincipled preparation. Hence, acceptable information of statistics and the suitable use of statistical assessments are significant. An suitable information approximately the simple statistical methods will go a long way in enlightening the research strategies and manufacturing superiority health research which can be operated for expressing the evidencebased strategies.

\section{REFERENCES}

1. Ali, Z., \& Bhaskar, S. (2016). Basic statistical tools in research and data analysis. Indian Journal of Anaesthesia. 60. 662. 10.4103/0019. 5049.190623.

2. Manikandan, S. (2011). Measures of central tendency: The mean. Journal of pharmacology \& pharmacotherapeutics. 2. 140-2. 10.4103/0976500X.81920.

3. Manikandan, S. (20111). Frequency distribution. J Phamacol Pharmacother; 2:54-6.

4. Gravetter, F.J., Wallnau, L.B. (2000). Statistics for the behavioral sciences. 5th ed. Belmont: Wadsworth - Thomson Learning;

5. Myles, P.S., Gin, T. (2000). Statistical Methods for Anaesthesia and Intensiven Care. Ist ed. Oxford: Butterworth Heinemann; p.

6. Senthilnathan, S. (2019). Usefulness of Correlation Analysis. SSRN Electronic Journal.

7. Arkkelin, D. (2014). Using Spss to Understand Research and Data Analysis. Valparaiso: Valparaiso University

8. Mehta, B. C., \& Kapoor, K. (2005). Fundamentals of Econometrics. Mumbai: Himalaya Publishing House

9. Zaid, M. A. (2015). Correlation and Regression Analysis; Statistical Economic and Research and Training Centre for islamic countries

10. Runhua., \& Conrad, S. (2009). Correlation and regression analysis. Ann Allergy Asthma Immunol. 103. S35-41.

11. Christof, S., Ke-Hai, Y. (2005). in Encyclopedia of Social Measurement.

12. Kabir, S. M. (2016). Methods of data collection

13. Ather, S., \& Balasundaram, N. (2009). Factor Analysis: Nature, Mechanism \& Uses in Social and Management Researches. Journal of Cost and Management Accountant, Bangladesh.

14. Gogtay, N., \& Thatte, U. (2017). Principles of Correlation Analysis. The Journal of the Association of Physicians of India, 65, 78-81.

15. Hauke, J., \& Kossowski, T. (2011). "Comparison of Values of Pearson's and Spearman's Correlation Coefficients on the Same Sets of Data," Quaestiones Geographicae, Sciendo, 30(2), 87-93, June.

16. Gogtay, N.J., \& Thatte, U.M. (2017). Statistics for Researcher Principles of Correlation Analysis. Journal of the Association of Physicians of India, $65,78-80$. 